\title{
Mesenchymal stem cell-tumor cell cooperation in breast cancer vasculogenesis
}

\author{
ŞERBAN COMŞA ${ }^{1}$, FELICIA CIUCULESCU ${ }^{2}$ and MARIUS RAICA ${ }^{1}$ \\ ${ }^{1}$ Department of Histology, Angiogenesis Research Center; ${ }^{2}$ Department of Physiology, \\ 'Victor Babeş' University of Medicine and Pharmacy, Timişoara, Romania
}

Received November 28, 2011; Accepted January 30, 2012

DOI: $10.3892 / \mathrm{mmr} .2012 .796$

\begin{abstract}
Mesenchymal stem cells (MSCs) are able to acquire endothelial-like characteristics but their involvement in regulating MSC vasculogenesis is more complex. MSCs are able to express endothelial markers when cultured in endothelial growth medium (EGM), proving their differentiation into endothelial-like cells. The aim of our study was to evaluate the capacity of the MCF-7 breast cancer cell line to stimulate the organization of regular MSCs and MSCs culture-expanded in EGM (MSCEs) into capillary-like structures and to assess the involvement of tumor-derived VEGF. We seeded MSCs and MSCEs on Matrigel in a Transwell two compartment culture system in the presence of VEGF, MCF-7 cells or their conditioned medium (CM). Both MSCs and MSCEs were CD31-negative, either in culture conditions, or in the Transwell system. MSCs had a clear tendency to organize in clusters and to form capillary-like structures, in the presence of VEGF or MCF-7 cells. MSCEs had a similar behavior, but their tendency to organize in clusters was lower. Neither MSCs nor MSCEs organized into capillary-like structures in the presence of MCF-7 CM, yet the tendency to organize in clusters was stronger in the MSCs. Following exposure both to EGM-2 alone and to EGM-2 supplemented with MSCs or MSCEs, the MCF-7 cells were present as adherent cells on the bottom of the lower wells, while the tendency to organize as single cells (and not in clusters) was more evident when MCF-7 cells were co-cultured with MSCs compared to the other conditions. Both breast cancer cells and VEGF stimulate MSCs and MSCEs to form capillary-like structures, indicating a role of tumorderived VEGF in modulating their recruitment into sites of pathological vasculogenesis. Preconditioning MSCs in EGM influenced their pattern of organization into capillary-like structures, but the potential changes in the molecular marker
\end{abstract}

Correspondence to: Dr Şerban Comşa, Department of Histology, Angiogenesis Research Center, 'Victor Babeş' University of Medicine and Pharmacy, Piaţa Eftimie Murgu 2, 300041 Timişoara, Romania E-mail: serbancomsa@yahoo.com

Key words: breast cancer, MCF-7, mesenchymal stem cells, vasculogenesis, VEGF profile for their 'switch' to the endothelial cell line remain to be evaluated.

\section{Introduction}

Mesenchymal stem cells (MSCs) are a subset of non-hematopoietic cells within the bone marrow stroma that have the ability to self renew and to differentiate into cells of multiple lineages (1). MSC differentiation to a vascular cell fate, such as endothelial cells or vascular smooth muscle cells (SMCs), is less well defined and more controversial (2).

It has been demonstrated that MSCs have the capability to acquire endothelial-like characteristics in pro-angiogenic environments (3), which allow them to be involved in angiogenic processes, including cell proliferation, migration, tube formation and vessel stabilization (4). It has also been reported that MSCs express endothelial markers when cultured in endothelial growth medium (EGM), proving their differentiation into endothelial-like cells (3).

MSCs are capable of differentiating into endothelial cells in vitro (5) and they can stimulate the formation of new blood vessels in vivo (3), through their functional vascular plasticity (6) and physical incorporation into the vasculature (7). MSCs have the ability to be recruited at active sites of angiogenesis and to promote a robust host-derived angiogenic response in vivo (4).

MSCs are able to participate in the production of vessels through their ability to migrate and generate capillary-like structures and these angiogenic properties are modulated through growth factor-mediated paracrine regulation (8).

Soluble factors, such as VEGF, play a role in inducing MSC migration, proliferation and differentiation into vascular cell types (9). VEGF involvement in regulating MSCs during vasculogenesis is less well understood and likely to be extremely complex (2). It has been demonstrated that MSCs can be induced to differentiate towards endothelial cells, following exposure to VEGF $(2,5,6)$. The potent angiogenic stimulant VEGF is known to regulate MSC mobilization and recruitment to sites of neovascularization, as well as directing the differentiation of MSCs to a vascular cell fate (7). It has also been demonstated that VEGF markedly enhances the formation of tube-like structures by MSCs, when cultivated in semi-solid medium.

Recent studies reveal that MSCs do not express VEGF receptors, but VEGF can promote MSC proliferation and 
migration by activating platelet-derived growth factor (PDGF) receptors in MSCs (10). VEGF-A/PDGFR signaling, especially during tissue hypoxia, is likely to be an important determinant in the recruitment and proliferation of MSCs (10). The recruitment of MSCs to sites of active tumor neovascularization and the differentiation towards endothelial cells is also predicted to involve VEGF-A-induced PDGFR 2 (2).

The mechanism by which MSCs contribute to tumor vessel formation is at least complex and not precisely known (7). MSCs can participate in active tumor angiogenic processes as they are massively recruited by both differentiated endothelial cells and cancer cells (4). Data indicate a role for MSCs in modulating the tumor microenvironment by its production of a large number of cytokines, growth factors and extracellular matrix proteins, expression of various cytokine and growth factor receptors, as well as by a (trans)-differentiation in endothelial-like and pericyte-like cells (7).

VEGF is a major angiogenic factor in breast tumor progression (11). In addition to stimulating breast tumor angiogenesis by a paracrine mechanism, VEGF also promotes breast cancer cell growth, survival, and invasion by an autocrine pathway (11). Since the VEGF gene is hypoxia responsive, it is thus tempting to suggest that reduced oxygen tension, such as that encountered within tumor masses, may provide the stimulus required to locally promote VEGF production by MSCs and to simultaneously initiate a potent host-derived angiogenic response (4).

MSCs in cancer stroma may play a pivotal role in modulating cancer cell proliferation, metastasis, and chemotherapy resistance (12). MSCs were shown to increase the rate of tumor spread and also to confer more invasive properties upon the circulating tumor cells (1). Interestingly, some authors report a universal downregulation of genes associated with proliferation in the breast cancer cells retrieved from co-culture with MSCs (13). By contrary, others suggest that MSC integration into growing breast cancer is critical for tumor growth (14).

MCF-7, an established human metastatic breast cancer cell line, was designed at the Michigan Cancer Foundation in 1973, from a pleural effusion and is the most commonly used breast cancer cell line worldwide (15).

Fierro et al (16) showed that changes were detected in the morphology, proliferative capacity and aggregation pattern of MCF-7 cells after co-culture with MSCs. On the other hand, MCF-7 cells express VEGF, which is critical for the induction of MSC migration by breast cancer cell-conditioned medium (14).

The aim of our study was to evaluate the capacity of MCF-7 breast cancer cell line to stimulate the organization of regular MSCs and MSCs culture-expanded in EGM (MSCEs) into capillary-like structures and to assess the involvement of tumor-derived VEGF in this process.

\section{Materials and methods}

Chemicals and reagents. The following materials were purchased from the indicated sources. Fluorescence-conjugated monoclonal antibody for CD31 (fluorescein isothiocyanate), insulin, Matrigel and glutamine were purchased from Sigma (Steinheim, Germany). Fluorescence-conjugated monoclonal antibodies for CD45 (phycoerythrin-Cy5), CD73 (phycoerythrin), CD90 (fluorescein isothiocyanate), and
CD105 (phycoerythrin), the basic fibroblast growth factor (bFGF) and human recombinant VEGF were from R\&D Systems, Inc. (Minneapolis, MN). Phosphate-buffered saline, penicillin-streptomycin solution, trypan blue stain $0.4 \%$ and trypsin EDTA (0.25\% solution) were from Gibco (Karlsruhe, Germany).

Cell sources. Human MSCs were isolated from the bone marrow of patients undergoing hip replacement surgery, after informed consent of the patients. The cells stained negatively for the hematopoietic marker CD45 and positively for the mesenchymal cell markers CD73, CD90 and CD105 as determined by flow cytometric analysis. Their pluripotency was confirmed by successful induction of osteoblastic, chondrogenic, and adipogenic differentiation upon addition of the respective media under standardized conditions.

MSCs were culture expanded and trypsinized when they reached $70-80 \%$ confluence. Cells were reseeded at $2-3 \times 10^{3} /$ $\mathrm{cm}^{2}$ in T75 flasks (Becton-Dickinson, Heidelberg, Germany) filled with $12 \mathrm{ml}$ of low-glucose Dulbecco's modified Eagle's medium (DMEM; Gibco, Paisley, UK) supplemented with 20\% fetal bovine serum (FBS; PAN Systems, Passau, Germany), $25 \mathrm{ng} / \mathrm{ml} \mathrm{bFGF}$ and $1 \%$ penicillin/streptomycin mix and incubated at $37^{\circ} \mathrm{C}$ in $5 \% \mathrm{CO}_{2}$.

Simultaneously, a part of the MSCs, entitled MSCEs, was cultured in endothelial cell growth medium-2 (EGM-2) from Lonza (Walkersville, MD). MSC and MSCE medium renewal was performed 2 times/week After 3 weeks, before using them in experiments, the cells were tested for their capacity to differentiate into endothelial cells, through determination of the endothelial marker CD31 by flow cytometric analysis.

MCF-7 cells (HTB-22) were purchased from ATCC (Wesel, Germany). Cells were seeded in T75 flasks at $1 \times 10^{6}$ cells/flask in low glucose DMEM containing 10\% FBS, 2 mM glutamine, $0.01 \mathrm{mg} / \mathrm{ml}$ insulin and $1 \%$ penicillin/streptomycin mix (MCF-7 medium) and incubated at $37^{\circ} \mathrm{C}$ in an atmosphere of $5 \% \mathrm{CO}_{2}$. The medium renewal was performed 2 times/ week, while cells were weekly passaged at a sub-cultivation ratio of 1:3.

Collection of conditioned medium. To detect the presence of chemokines potentially mediating any paracrine effects of MCF-7 cells on MSCs/MSCEs, conditioned medium (CM) from MCF-7 cells was collected and analyzed in the capillarylike assay. CM refers to medium that was incubated in the presence of cells for $24 \mathrm{~h}$. This medium was then harvested and centrifuged twice (1200 rpm, $5 \mathrm{~min}$ ) to avoid the presence of MCF-7 cells and then it was used in the experiment.

The capillary-like assay. For the capillary-like assay, MSCs and MSCEs were trypsinized, washed with PBS and resuspended in EGM-2 at $37^{\circ} \mathrm{C}$, at a density of $1 \times 10^{5}$ cells $/ \mathrm{ml}$. The MCF-7 cells were isolated from their medium through centrifugation (1200 rpm, $5 \mathrm{~min}$ ) and then they were resuspended in fresh MCF-7 medium at a density of $1 \times 10^{6}$ cells $/ \mathrm{ml}$.

A 24-well Transwell system with polycarbonate $0.4-\mu \mathrm{m}$ pore membranes (Transwell insert diameter $6.5 \mathrm{~mm}$, insert membrane growth area $0.33 \mathrm{~cm}^{2}$ ) from Corning Life Sciences (Wiesbaden, Germany) was used. Matrigel was added to the upper wells according to the manufacturer's instructions and it 


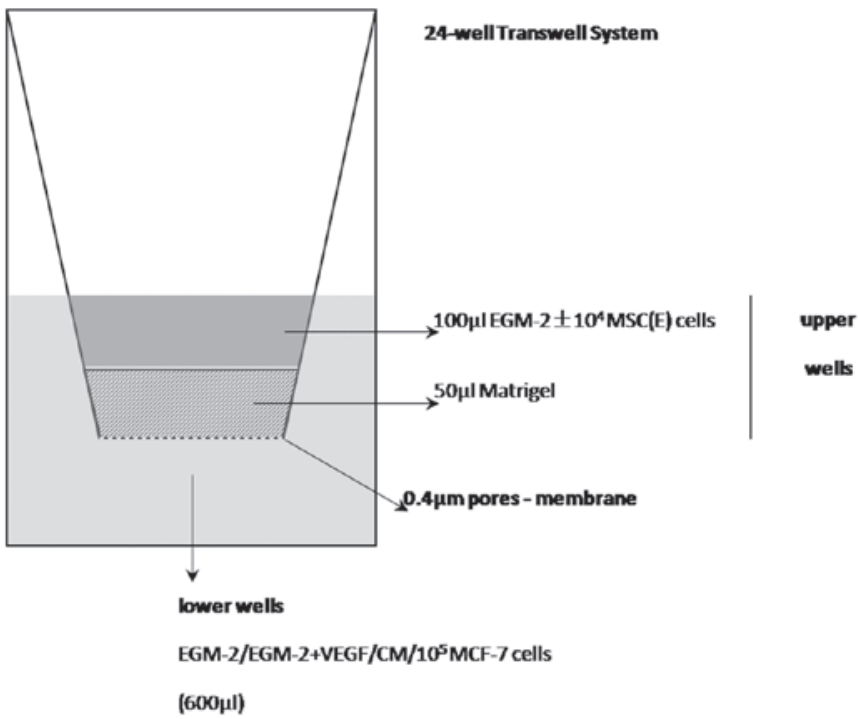

Figure 1. Schematic presentation of the capillary-like assay. The lower wells were filled with EGM-2 alone/EGM-2 supplemented with VEGF (50 ng/ml)/ conditioned medium (CM) of the MCF-7 cell line/10 $\mathrm{MCF}-7$ cell suspension $(600 \mu 1)$. The upper wells were coated with Matrigel $(50 \mu 1)$ and EGM-2 (100 $\mu 1$ ) supplemented or not with MSC/MSCE cells added over it. The upper and the lower wells were separated by a $0.4-\mu \mathrm{m}$ pore size membrane which did not allow cell transmigration.

was allowed to dry on the membrane surface in a bacteriological hood (3-4 h). The lower wells were then filled with EGM-2 alone, EGM-2 supplemented with VEGF, CM or MCF-7 cell suspension, at a total volume of $600 \mu \mathrm{l}$ (all the media were prewarmed at $\left.37^{\circ} \mathrm{C}\right)$. Cell suspensions (100 $\mu$ l EGM-2) containing $10^{4}$ MSCs or MSCEs, but also EGM-2 alone, were pipetted on the Matrigel-coated surface from the upper wells and a plastic cover was then placed on the top of the wells. The 24-well Transwell system was placed into a humidified incubator at $5 \% \mathrm{CO}_{2}$ at $37^{\circ} \mathrm{C}$.

After $48 \mathrm{~h}$, the upper wells were removed from the Transwell system and the medium was carefully discharged from the upper wells, leaving the Matrigel in place. The upper portion of the Transwell inserts was rapidly sliced off using a heated scalpel, leaving the stained membrane and 3-4 mm of plastic insert attached to it. Evaluation of the potential capillary-like structures formed by MSCs/MSCEs in Matrigel was performed using an Olympus CK-2 upright microscope, at 40/100-fold magnification. The bottom of the lower wells containing MCF-7 cells was also evaluated, in order to describe the arrangement of these cells. Images of representative fields were captured to document the findings using a digital camera.

Immunofluorescence. In order to evaluate the CD31 status in MSCs and MSCEs from the capillary-like assay, we performed immunofluorescence staining of the cells from the upper wells. The samples were fixed in $4 \%$ paraformaldehyde in PBS for $15 \mathrm{~min}$ and then they were incubated with FITC monoclonal anti-CD31 antibody in 1\% BSA in PBS in a humidified chamber for $1 \mathrm{~h}$ at $37^{\circ} \mathrm{C}$. In order to stain DNA, cells were incubated on $1 \mu \mathrm{g} / \mathrm{ml}$ DAPI for $1 \mathrm{~min}$. At the end of the immunofluorescence protocol the samples were evaluated using an epifluorescence microscope (Axio-skop 2 Zeiss) and photos were taken for documentation.

\section{Results}

To establish whether MSCs could differentiate into endothelial-like cells following exposure to endothelial media we culture-expanded them in EGM-2. As controls, we used MSCs maintained in DMEM-10\% FBS. After $24 \mathrm{~h}$ of culture, MSCs and MSCEs presented a similar morphology, but after 3 weeks we noticed that the morphology of confluent MSCEs was different from that of MSCs. In addition, after a 3-week culture in the specified media, expression of CD31 was absent both in the MSCs and MSCEs.

We tested the ability of different media to induce MSCs or MSCEs to organize in capillary-like structures using a Boyden chamber (Transwell) assay. The cells were seeded into the upper wells onto Matrigel and incubated with plasma, VEGF, CM of MCF-7 cells or MCF-7 cell suspension, in the lower wells (Fig. 1). The potential capillary-like structures were evaluated after $48 \mathrm{~h}$ of incubation.

MSCs had a clear tendency to organize in clusters and to form capillary-like structures in the presence of VEGF. Similar results were obtained in the presence of the MCF-7 cells. MSCEs also organized into capillary-like structures in the presence of both VEGF and the MCF-7 cell suspension, but these structures were more unclear, maybe due to their lower tendency to organize in clusters (Fig. 2). Neither MSCs nor MSCEs organized in clear capillary-like structures when they were exposed to MCF-7 CM.

MSCs also developed long and thin prolongations interconnecting each other following exposure to VEGF. A similar pattern was noted when MSCs were co-cultured with MCF-7 cells (Fig. 3). MSCEs developed much shorter prolongations comparing to MSCs in the corresponding wells.

We performed immunofluorescence in order to evaluate the CD31 expression in the cells from the upper well after the 48 h-incubation with VEGF, CM and the MCF-7 cell suspension in the lower wells. Independent of the content of the corresponding lower wells, neither MSCs nor MSCEs expressed CD31.

We also evaluated the behavior of the MCF-7 cells from the lower wells following exposure to EGM-2 alone or supplemented with MSCs or MSCEs. The MCF-7 cells were present as adherent cells on the bottom of the lower wells in all of the conditions, yet their tendency to organize as single cells (and not in clusters) was more evident when MCF-7 cells were co-cultured with MSCs compared to the other conditions (Fig. 4).

\section{Discussion}

In order to evaluate whether MSCs differentiate into endothelial-like cells and express endothelial markers when cultured in endothelial growth medium (EGM) as previously reported (3), we culture-expanded them both in their specific medium and in EGM-2. Although after $24 \mathrm{~h}$ of culture, MSCs and MSCEs presented a similar morphology, after 3 weeks, similar to the results of Liu et al (3), differences in the morphology of confluent MSCEs, as compared to MSCs, were noted. After a 3-week culture in their respective media, expression of CD31 was absent both in MSCs and MSCEs. Opposite to this result, Liu et al (3) concluded that CD31 expression was increased 

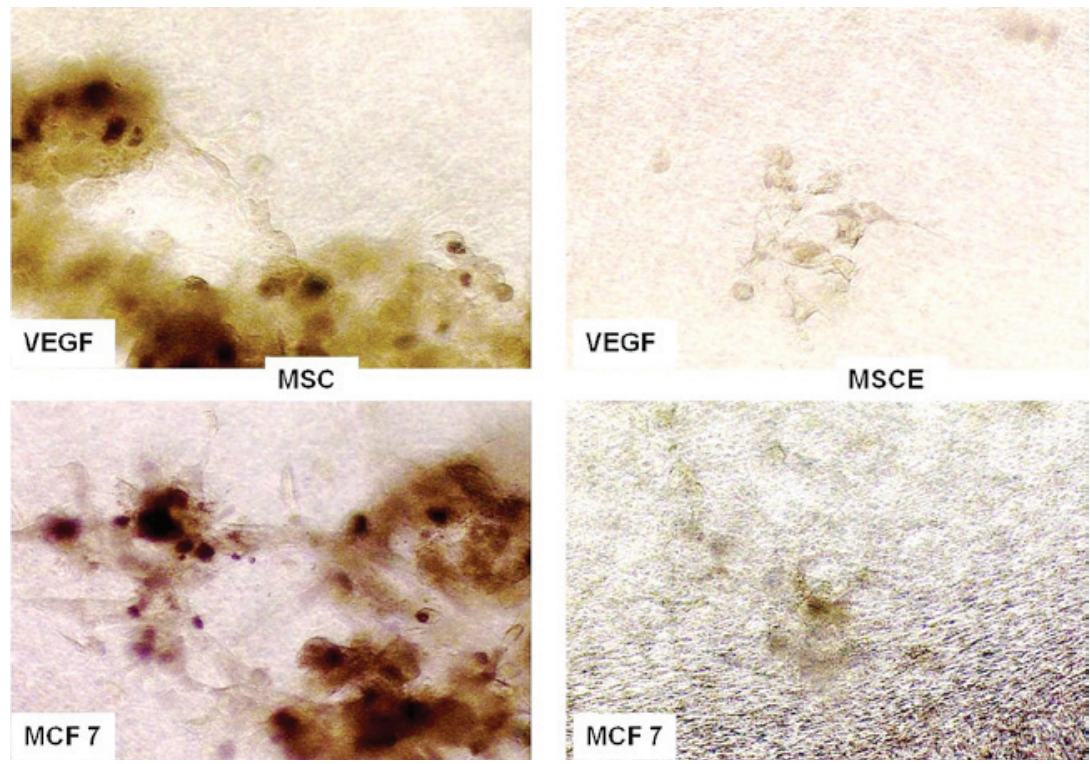

Figure 2. The capillary-like structures formed by MSCs and MSCEs in the presence of VEGF or MCF-7 cell suspension. MSCs had a higher tendency to organize in clusters compared to MSCEs, and the capillary-like structures they organized were much more evident than those formed by MSCEs. The textboxes refer to the content of the lower wells of the Transwell system (magnification, x100).
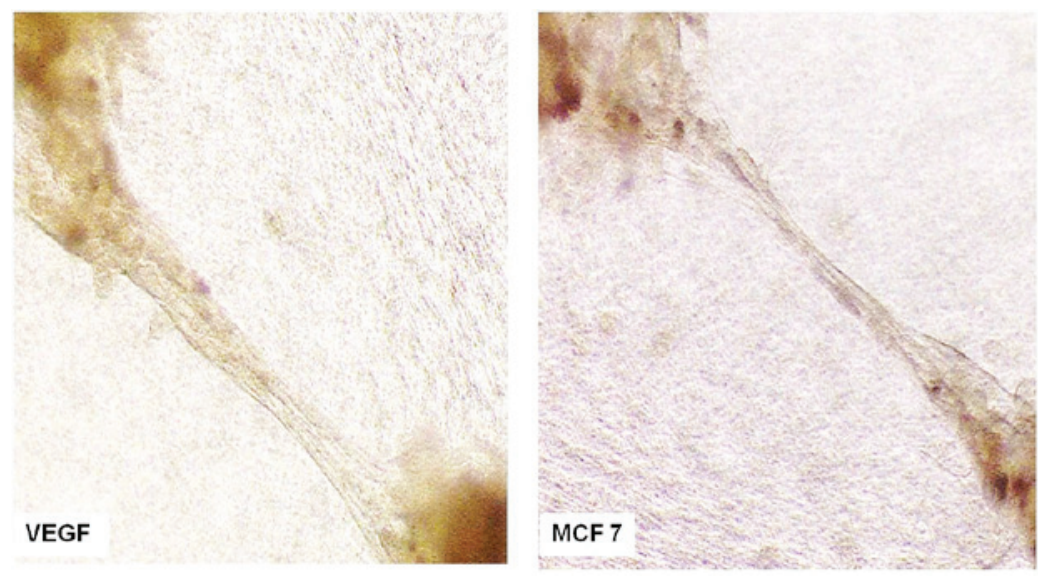

Figure 3. MSCs developed long and thin prolongations interconnecting each other, in the presence of VEGF or MCF-7 cells. The textboxes refer to the content of the lower wells of the Transwell system (magnification, x100).
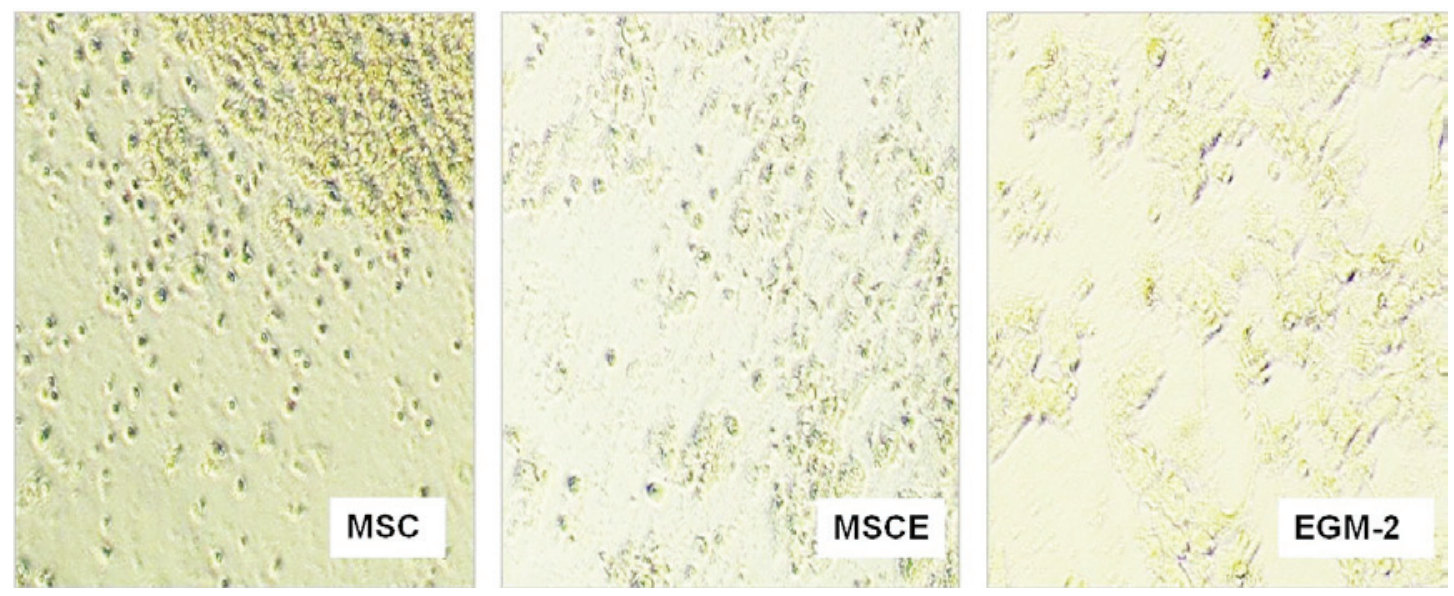

Figure 4. MCF-7 cells from the lower wells after exposure to EGM-2 supplemented or not with MSCs or MSCEs. The cells were adherent to plastic in all three conditions. Their tendency to organize as single cells was quite evident after exposure to MSCs. In co-culture with MSCEs, the MCF-7 cells were present both as single cells and in clusters, while after exposure to EGM-2 alone, the majority of cells were organized in clusters and the single cells were very rare. The textboxes refer to the content of the upper wells of the Transwell system (magnification, x100). 
in MSCEs as compared with MSCs. Notably, Oswald et al (5) found, that following exposure to VEGF $(50 \mathrm{ng} / \mathrm{ml})$ for 7 days, MSCs acquired major characteristics of mature endotheliallike expression of vWF, VEGF receptors 1 and 2, VE- cadherin and VCAM-1, while they did not express CD31 and CD34.

We described the conditions that allow the migration and organization of MSCs and MSCEs into capillary-like structures. Annabi et al (4) suggested that MSCs possess an inherent capacity to rapidly form in vitro tube-like structures when cultured on 3D-Matrigels, but this was not the case in our study, as neither MSCs, nor MSCEs formed such structures when they were suspended in EGM-2 and cultured for $48 \mathrm{~h}$ in Matrigel. In addition, Al-Khaldi et al (6) sustain that although MSCs unambiguously become CD31 ${ }^{+}$and $\mathrm{VEGF}^{+}$ spontaneously in vivo without the need of exogenous angiogenic factors, MSCs cultured ex vivo in Matrigel do not adopt this phenotype spontaneously.

We evaluated the potential of VEGF $50 \mathrm{ng} / \mathrm{ml}, \mathrm{CM}$ and MCF-7 suspension to induce the organization of MSCs/MSCEs into capillary like structures. Annabi et al (4) suggested that MSCs may share some common molecular and cellular phenotypes with differentiated endothelial cells in regards to the tumor microenvironment that may actively regulate their involvement in angiogenesis. We demonstrated, in a Boyden chamber (Transwell) assay, that MSCs had a clear tendency to organize into clusters and to form capillary-like structures in Matrigel both in the presence of VEGF and the MCF-7 cell suspension, indicating a role for the tumor-derived VEGF in this process. Similar results were obtained by Annabi et al (4) who concluded that MSCs are able to generate three-dimensional capillary-like structures on Matrigel and this property appears to be upregulated by tumor-derived growth factors. In addition, Oswald et al (5) sustain that MSCs showed a substantial formation of capillary structures when cultivated in the presence of VEGF.

Although Liu et al (3) proved that the differentiation of MSCs into MSCEs do not impair or increase their ability to induce new blood vessels, we noted that MSCEs organized into unclear capillary-like structures compared to MSCs, both in the presence of VEGF and the MCF-7 cell suspension. In addition, the study of Liu et al (3) revealed, after a direct comparison of MSCs and MSCEs on Matrigel, that in the absence of VEGF the MSCEs were able to form cord- or tube-like structures in a shorter time compared to MSCs. They also concluded that MSCEs induced the generation of functional blood vessels in vivo and were incorporated into these blood vessels.

Although it has been demonstrated that capillary-like structure formation is strongly stimulated by CM isolated from different cancer cell lines (4), neither MSCs nor MSCEs organized in clear capillary-like structures when they were exposed to $\mathrm{MCF}-7 \mathrm{CM}$.

Notably, MSCEs developed much shorter prolongations comparing to MSCs following exposure to VEGF or the MCF-7 cell suspension. To our knowledge, this is the first study to report these morphological differences between MSCs and MSCEs following exposure to VEGF or a tumoral environment in a Transwell assay.

Since Oswald et al (5) demonstrated that after a 7-day exposure to VEGF, MSCs transdifferentiated into CD31- negative endothelial-like cells, the lack of CD31 expression in MSCs after a 48-h exposure to VEGF, CM and the MCF-7 cell suspension could be explained by the fact that this marker is later expressed in endothelial maturation. In addition, it is suggested that elongation of the differentiation time would probably also lead to an upregulation of this marker. A controversial aspect is that MSCEs did not express CD31 either in the conditions presented above, while Liu et al (3) sustains that CD31 expression was increased in MSCEs as compared with MSCs, after a 3-week culture in the specified media.

Relatively little is known concerning the characteristics of MSCs in solid tumors. It remains unknown whether MSCs are located in primary breast cancer tissues and how they participate in tumor proliferation (12). As an important component of the tumor microenvironment, MSCs can interact with breast cancer cells via direct contact or paracrine regulatory mechanisms (12). MSCs were shown to increase the rate of tumor spread and also to confer more invasive properties upon circulating tumor cells (1). Interestingly, most of the MCF-7 cells cultured in the presence of EGM-2 supplemented or not with MSCs/MSCEs adhered to the bottom of the wells, while in culture flasks most of them were non-adherent cells. Following exposure to MSCs, MSCEs and EGM-2 alone, their tendency to organize as single cells was more evident when MCF-7 cells were co-cultured with MSCs compared to the other conditions. In this case, the majority of the MCF-7 cells were observed on the bottom of the wells as single cells, without organizing into clusters. Similar results were obtained by Hombauer and Minguell (17), who concluded that more than $80 \%$ of MCF-7 cells co-cultured with MSCs were present as single cells lying close together without any evidence of direct cell-cell contacts. In conclusion, both MCF-7 cells and VEGF stimulate MSCs and MSCEs to form capillary-like structures, indicating a role of tumor-derived VEGF in modulating their recruitment into sites of tumor vasculogenesis in breast cancer. Preconditioning MSCs in EGM appear to influence their pattern of organization into capillarylike structures, but the potential corresponding changes in the profile of molecular markers require further elucidation in order to define their 'switch' to the endothelial cell line.

\section{Acknowledgements}

We are grateful to Dr Anca Maria Cîmpean, Dr Erika Deak, Dr Giani Oancea, Dr Raluca Ceauşu, Dr Puşa Gaje, Dr Dragoş Izvernariu, Reinhard Henschler and Victoria Lang for the excellent technical and scientific support. This work was supported by grant IDEI 345/2011 of the National Research Council of the Romanian Ministry of Education and Research.

\section{References}

1. Molloy AP, Martin FT, Dwyer RM, et al: Mesenchymal stem cell secretion of chemokines during differentiation into osteoblasts, and their potential role in mediating interactions with breast cancer cells. Int J Cell 124: 326-332, 2009.

2. Ball SG, Shuttleworth CA and Kielty CM: Mesenchymal stem cells and neovascularization: role of platelet-derived growth factor receptors. J Cell Mol Med 11: 1012-1030, 2007.

3. Liu JW, Mai G, Lambert J and Fish RJ: Characterization of endothelial-like cells derived from human mesenchymal stem cells. J Thromb Haemost 5: 826-834, 2007. 
4. Annabi B, Naud E, Lee YT, Eliopoulos N and Galipeau J: Vascular progenitors derived from murine bone marrow stromal cells are regulated by fibroblast growth factor and are avidly recruited by vascularizing tumors. J Cell Biochem 91: 1146-1158, 2004.

5. Oswald J, Boxberger S, Jørgensen B, et al: Mesenchymal stem cells can be differentiated into endothelial cells in vitro. Stem Cells 22: 377-384, 2004.

6. Al-Khaldi A, Eliopoulos N, Martineau D, Lejeune L, et al: Postnatal bone marrow stromal cells elicit a potent VEGFdependent neoangiogenic response in vivo. Gene Ther 10: 621-629, 2003.

7. Roorda BD, Elst A, Kamps WA and de Bont ES: Bone marrow-derived cells and tumor growth: contribution of bone marrow-derived cells to tumor micro-environments with special focus on mesenchymal stem cells. Crit Rev Oncol Hematol 69: 187-198, 2009

8. Annabi B, Lee YT, Turcotte S, et al: Hypoxia promotes murine bone-marrow-derived stromal cell migration and tube formation. Stem Cells 21: 337-347, 2003.

9. Huang NF and Li S: Mesenchymal stem cells for vascular regeneration. Regen Med 3: 877-892, 2009.

10. Ball SG, Shuttleworth CA and Kielty CM: Vascular endothelial growth factor can signal through platelet-derived growth factor receptors. J Cell Biol 177: 489-500, 2007.
11. Guo P, Fang Q, Tao HQ, et al: Overexpression of vascular endothelial growth factor by MCF-7 breast cancer cells promotes estrogen-independent tumor growth in vivo. Cancer Res 63: 4684-4691, 2003.

12. Yan XL,Fu CJ, Chen L, et al: Mesenchymal stem cells from primary breast cancer tissue promote cancer proliferation and enhance mammosphere formation partially via EGF/EGFR/Akt pathway. Breast Cancer Res Treat: May 17, 2011 (E-pub ahead of print).

13. Martin FT, Dwyer RM, Kelly J, et al: Potential role of mesenchymal stem cells (MSCs) in the breast tumour microenvironment: stimulation of epithelial to mesenchymal transition (EMT). Breast Cancer Res Treat 124: 317-326, 2010.

14. Ritter E, Perry A, Yu J, Wang T, Tang L and Bieberich E: Breast cancer cell-derived fibroblast growth factor 2 and vascular endothelial growth factor are chemoattractants for bone marrow stromal stem cells. Ann Surg 247: 310-314, 2008.

15. Burdall SE, Hanby AM, Lansdown MR and Speirs V: Breast cancer cell lines: friend or foe? Breast Cancer Res 5: 89-95, 2003.

16. Fierro FA, Sierralta WD, Epuñan MJ and Minguell JJ: Marrowderived mesenchymal stem cells: role in epithelial tumor cell determination. Clin Exp Metastasis 21: 313-319, 2004.

17. Hombauer $\mathrm{H}$ and Minguell J: Selective interactions between epithelial tumour cells and bone marrow mesenchymal stem cells. Br J Cancer 82: 1290-1296, 2000. 